\title{
ОСОБЕННОСТИ ПРЕПОДАВАНИЯ СПИРОМЕТРИЧЕСКОГО МЕТОДА ВРАЧАМ В ПОСЛЕДИПЛОМНОМ ОБУЧЕНИИ
}

\begin{abstract}
Метод оценивания вентиляционной способности лёгких - спирометрия (или спирография) - является базовым в обследовании больного с предполагаемым или установленным заболеванием респираторного тракта аналогично с измерением артериального давления и регистрацией электрокардиограммы при исследовании сердечно-сосудистой системы [1, 2]. Спирометрия является решающим исследованием в диагностике и лечении хронической обструктивной болезни легких [3] и бронхиальной астмы $[4,5]$. Интерпретация полученных результатов входит в перечень профессиональных навыков пульмонологов, терапевтов, врачей функциональной диагностики, причем последние должны уметь выполнять спирометрию. Изучение методов обследования больных с патологией органов дыхания предусмотрено рабочими программами циклов повышения квалификации, стажировки, профессиональной переподготовки по специальностям «Терапия», «Пульмонология», а также Модуля 3 повышения квалификации в системе непрерывного медицинского образования по специальности «Терапия». Перечисленные дисциплины преподаются на кафедре терапии факультета интернатуры и последипломного образования (ФИПО) им. проф. А.И. Дядыка Государственной образовательной организации высшего профессионального образования «Донецкий национальный медицинский университет им. М. Горького».
\end{abstract}

\section{ЦЕЛЬ РАБОТЫ}

Описание некоторых вопросов преподавания метода спирометрии слушателям на кафедре терапии ФИПО им. проф. А.И. Дядыка, что может быть полезным для обучения врачей разных специальностей (функциональной диагностики, семейных врачей, терапевтов, пульмонологов).

\section{История создания метода спирографии}

Во вступительной части занятия преподаватель кратко рассказывает историю создания и дальнейшие этапы развития спирографии. Первые сообщения об изобретенном приборе для измерения объемов выдыхаемого воздуха опубликовал в 1846 г. британский врач J. Hutchinson [6]. Использование описываемого метода позволило определить ряд основных показателей в исследовании функции внешнего дыхания (ФВД), которые остаются актуальными в наше время. В 1925 г. швейцарский физиолог и инженер A. Fleisch внедрил в практику прибор для регистрации объемной скорости потока воздуха в процессе дыхания [7]. Разработанный принцип измерения объемной скорости потока по перепаду давления используется и в современных приборах. В 1951 г. результаты первого исследования, показавшего высокую диагностическую ценность одновременного изучения скорости потока и объемов дыхания, опубликовал H. Dayman, который детально описал кривые поток-объем у пациентов с различной патологией органов дыхания и физиологически обосновал взаимосвязь этих величин [8]. В 1950-х годах B. Wright создал и ввел в практику первый портативный спирометр и пикфлоуметр [9].

Начиная с 1980-х годов в практику стали внедряться аппараты с микропроцессорами и цифровыми технологиями обработки сигналов. С этого времени метод завоевал высокое доверие врачей различных специальностей, поскольку обладает рядом преимуществ: достоверностью получаемых результатов, высокой информативностью, неинвазивностью и безвредностью (отсутствие лучевой нагрузки), относительной простотой и возможностью многократного выполнения в любое время. Непрерывное совершенствование спирометрической аппаратуры сделало этот метод диагностики заболеваний ор-

(c) А.А. Калуга, Г.Г. Тарадин, И.В. Ракитская, К.Э. Могилевская, О.А. Приколота, И.С. Маловичко, 2021 ( ) Университетская Клиника, 2021 
ганов дыхания незаменимым в современном мире доказательной медицины.

\section{ОСНОВНАЯ ЧАСТЬ}

Следующим этапом семинара является обсуждение анатомии бронхолегочной системы (строение верхних и нижних дыхательных путей), основ физиологии (функциональной системы дыхания, акта дыхания) и патофизиологии внешнего дыхания. Преподаватель совместно с обучающимися разбирает типы дыхания (внешнее, тканевое), процессы дыхания (транспорт газов кровью от легких к органам), механизмы газообмена в легких, понятие, классификацию и патофизиологические особенности дыхательной недостаточности. Затем дается краткая характеристика типов нарушений вентиляционной способности легких (обструктивный, рестриктивный и смешанный) и механизмов их развития.

Подготовка к исследованию, технические особенности аппаратуры

Важным разделом разбираемой темы является изучение показаний и противопоказаний к проведению спирометрии.

Показания к проведению спирометрии [10, 13].

Диагностика:

- Установление причины респираторных жалоб больного

- Оценка влияния болезни на функцию легких

- Обследование лиц с высоким риском легочных заболеваний

- Оценка прогноза заболевания

Наблюдение:

- Оценка эффективности лечения

- Мониторирование течения заболевания

- Наблюдение за популяцией лиц, подвергающихся воздействию

неблагоприятных факторов

- Наблюдение за состоянием легочной системы среди лиц, принимающих препа-раты, обладающие легочной токсичностью

Экспертная оценка трудоспособности:

- Обследование больного перед началом реабилитации

- Оценка рисков как части экспертной оценки нетрудоспособности

- Экспертная оценка состояния здоровья по другим юридическим поводам

Общественное здоровье:

- Эпидемиологические исследования

- Расчет должных значений спирометрических параметров

- Клинические исследования
Спирометрия практически не имеет абсолютных противопоказаний, но маневр форсированного выдоха следует с осторожностью выполнять у больных с развившимся пневмотораксом и первые 2 нед после его разрешения, в первые 2 нед после инфаркта миокарда, офтальмологических и полостных операций, при выраженном сохраняющемся кровохаркании, наличии аневризмы любой локализации или подозрении на нее и тяжелой бронхиальной астме [10-12].

Детальное изучение требований к оснащению кабинета, технических аспектов спирометрии (основных типов спирометров, требований, предъявляемых к аппаратуре, правил калибровки) предусмотрено для специалистов, непосредственно выполняющих метод (врачей функциональной диагностики) [13]. Для них обоснованным является не только теоретическое обсуждение процедуры калибровки, но и отработка указанного процесса в виде практического навыка непосредственно на аппарате с использованием калибровочного шприца. Калибровка - процедура, во время которой устанавливается связь между параметрами потоков и объемов, рассчитанными сенсором, и реальными величинами и которая необходима для проверки точности работы прибора и в случае необходимости коррекции его показаний. Следует обратить внимание слушателей на то, что, помимо этого, существует процедура проверки калибровки, во время которой исследователь удостоверяется, что спирометр по-прежнему находится в пределах требуемых параметров (+3\% от установленного объема калибровочного шприца) [13]. Коррекция результатов проводится в большинстве типов спирометров автоматически с помощью специальных систем ATPS (ambient temperature pressure saturated = лабораторные условия: температура и атмосферное давление окружающей среды при полном насыщении водяным паром) и ВТРS (body temperature pressure saturated = условия организма: температура тела, окружающее давление и полное насыщение водяным паром), настраивающих работу аппарата приведением состояния газа к различным условиям по влажности воздуха, давлению и температуре [10]. Это связано с тем, что температура и влажность воздуха в легких отличаются от температуры окружающей среды. Система BTPS coответствует условиям в легких и в выдыхаемом воздухе непосредственно сразу после выдоха, ATPS - охладившемуся до внешней температуры выдыхаемому воздуху [5]. Калибровка проводится ежедневно, с использованием калибровочного шприца заданного объема с разной скоростью движения поршня [11]. 
В связи с тем, что на исследование ФВД пациентов направляют в основном врачи- клиницисты (терапевты, пульмонологи, семейные врачи), они должны обладать знаниями по подготовке пациента к исследованию. Поэтому на занятии детально оговариваются рекомендации для пациента, которому планируется проведение спирографии: воздержание от курения минимум за 3-4 часа до исследования, исключение физической нагрузки не менее чем за 30 мин до него. У больного следует уточнить информацию о приеме лекарств, влияющих на бронхиальную проходимость, и оговорить необходимость исключения их приема в соответствии с фармакокинетикой препарата перед процедурой, если это возможно.

\section{Этапы проведения и оцениваемые парамет- ры I}

После изучения вышеперечисленных разделов следует приступить к разбору этапов проведения спирометрии - запись спокойного дыхания, маневры жизненной емкости легких (ЖЕЛ), форсированной жизненной емкости легких (ФЖЕЛ) и маневр максимальной вентиляции легких (МВЛ). Необходимо определить критерии качества и ошибки при выполнении различных маневров [5, 13, 14], а также обсудить наиболее часто встречаемые причины некорректных результатов (утечка воздуха из-за неполного смыкания губ, неправильное наложение носового зажима, закрытие просвета мундштука языком, задержка дыхания на высоте вдоха, кашель). Разница в объемах при выполнении дыхательных маневров не должна превышать 5\% или 150 мл. В противном случае исследование необходимо повторить.

Врач, получающий заключение с результатами выполненной спирографии, должен обладать достаточными знаниями основных параметров, что поможет ему правильно оценить ФВД, наличие, степень выраженности и тип нарушения вентиляционной способности легких, установить диагноз, назначить необходимое лечение, в последующем оценить его эффективность. Для получения подобных знаний на занятии со слушателями ФИПО разбираются основные параметры, определяемые в ходе исследования, и их отклонения от нормы.

К ним относят:

- дыхательный объем (ДО);

- минутный объем дыхания (МОД);

- резервный объем вдоха и выдоха (РОвд, РОвыд);

- ЖЕЛ;

- ФЖЕЛ;

- объем форсированного выдоха за первую секунду (ОФВ1);
- индекс Тиффно, определяемый как соотношение ОФВ1/ЖЕЛ;

- индекс Генслера - ОФВ1/ФЖЕЛ

- среднюю объемную скорость на уровне выдоха от 25\% до 75\% ФЖЕЛ (СОС25-75);

- мгновенные скоростные показатели (MOC25-75);

- пиковую объемную скорость;

- в случае необходимости - МВЛ.

Также вводятся понятия оценки эффективности вентиляции, скорость потребления кислорода организмом и термин мертвого пространства (объем воздуха, не участвующий в газообмене). Важным является то, что в норме ФЖЕЛ на 100150 мЛ меньше ЖЕЛ. При обструктивных заболеваниях разница может достигать 300-400 мл. Следует обратить внимание на то, что факт обструкции устанавливается при снижении ОФВ1/ ФЖЕЛ менее 70\%, а степень обструкции - по уровню снижения ОФВ1 от должных величин.

Далее обучающимся объясняют, что нормальные значения должных величин можно определить согласно наиболее известным и широко применяемым системам нормативов Р.Ф. Клемента и соавт. [15] для лиц моложе 70 лет с модификациями для детей [16], R.J. Knudson et al. [17] для лиц старше 70 лет, отклонений этих параметров от нормы. В таблице 1 приведены норма и градации отклонений показателей внешнего дыхания [18].

Существующие шкалы должных величин могут различаться по числу параметров. Каждая система имеет свои ограничения не только по возрасту, но и по росту и весу. В связи с этим при оценке спирометрических показателей нужно учитывать, что норма одного и того же параметра может быть различна в зависимости от выбранной шкалы. В таблице 2 представлены допустимые диапазоны возраста и роста в указанных системах.

Во время обсуждения значений и различных вариантов кривых поток-объем и объем-время при рестриктивных и обструктивных нарушениях преподаватель одновременно использует визуальное представление различных вариантов графиков и кривых как дополнение к разбираемому материалу. Подобный подход, совмещающий вербальный и зрительно-иллюстративный методы обучения, обычно вызывает большой интерес у аудитории и помогает лучше запомнить изучаемый материал.

Следует оговорить, что уровень бронхиальной проходимости на основании данных кривой поток-объем в настоящий момент не определяют. Кроме того, необходимо подчеркнуть, что обструкция констатируется, когда индекс ОФВ1/ ФЖЕЛ менее 70\%, только при условии сохранен- 
Таблица 1.

Границы нормы и градации отклонения показателей внешнего дыхания по Л.Л. Шику, Н.Н. Канаеву [18].

\begin{tabular}{lccccc}
\hline \multirow{2}{*}{ Показатели } & Норма, \% & Условная норма, \% & \multicolumn{3}{c}{ Изменения \% } \\
\cline { 4 - 6 } & & & Умеренные & Значительные & Резкие \\
\hline \hline ЖЕЛ & $>90$ & $89-85$ & $84-70$ & $69-50$ & $<50$ \\
\hline ОФВ & $>85$ & $84-75$ & $74-55$ & $54-35$ & $<35$ \\
\hline ОФВ $_{1} /$ ЖЕЛ & $>70$ & $70-65$ & $64-55$ & $54-40$ & $<40$ \\
\hline ОФВ $/$ ФЖЕЛ & $>70$ & $69-65$ & $64-55$ & $54-40$ & $<40$ \\
\hline МОС 25\% жен. & $>80$ & $80-67,2$ & $67,1-41,8$ & $41,7-33,3$ & $<33,3$ \\
МОС 25\% муж. & $>81,6$ & $81,6-69,8$ & $69,7-52,8$ & $52,7-45,9$ & $<35,9$ \\
\hline МОС 50\% жен. & $>77$ & $77-62$ & $62-32$ & $32-22$ & $<22$ \\
МОС 50\% муж. & $>76$ & $76-60$ & $60-30$ & $30-20$ & $<20$ \\
\hline МОС 75\% жен. & $>72$ & $72-54$ & $53-41$ & $40-27$ & $<27$ \\
МОС 75\% муж. & $>72$ & $72-55$ & $54-41$ & $40-23$ & $<23$ \\
\hline
\end{tabular}

Допустимые диапазоны возраста и роста пациентов в некоторых системах

Таблица 2. должных величин показателей функции внешнего дыхания [4].

\begin{tabular}{|c|c|c|c|}
\hline \multirow{2}{*}{ Система } & \multirow{2}{*}{ Пол } & \multicolumn{2}{|c|}{ Допустимый диапазон } \\
\hline & & Возраст, лет & $\mathrm{Poct}_{3}$ см \\
\hline \multirow{4}{*}{ Р.Ф. Клемент и др. } & \multirow{2}{*}{ Мужчины } & $6-17$ & $100-199$ \\
\hline & & $18-70$ & $149-203$ \\
\hline & \multirow{2}{*}{ Женщины } & $6-17$ & 100-199 \\
\hline & & $18-70$ & $140-185$ \\
\hline \multirow{6}{*}{ R.J. Knudson et al. } & \multirow{3}{*}{ Мужчины } & $6-11$ & $110-155$ \\
\hline & & $12-25$ & $138-200$ \\
\hline & & $26-85$ & $151-200$ \\
\hline & \multirow{3}{*}{ Женщины } & $6-10$ & $105-148$ \\
\hline & & $11-20$ & $130-185$ \\
\hline & & $21-85$ & $144-180$ \\
\hline
\end{tabular}

ной ФЖЕЛ [10]. ЕслИ ФЖЕЛ больше ЖЕЛ, то это считается ошибкой и исследование необходимо повторить, либо величину ФЖЕЛ принимать за ЖЕЛ. В случае снижения ЖЕЛ, а следовательно, и ФЖЕЛ, необходимо провести бодиплетизмографию для исключения/подтверждения истинных рестриктивных нарушений.

Для повышения интереса слушателей к изучаемой теме во время проводится разбор клинического случая с обсуждением полученных результатов спирографии и возможных рекомендаций по лечению и дальнейшей тактике ведения конкретного пациента.

\section{Бронходилатационный и бронхоконстрик- торный тесты}

Заключительным этапом обследования пациента является проведение бронходилатационного (БДТ) и бронхоконстрикторного (БКТ) тестов. Цели, особенности их выполнения подробно разбираются со слушателями ФИПО. БДТ необходим для оценки обратимости ОФВ1 после ингаляции бронхорасширяющих препаратов в качестве «золотого» стандарта установления обратимости обструкции.

Противопоказаний к проведению БДТ нет, кроме случаев, когда противопоказано проведение самой спирометрии или есть непереносимость бронхорасширяющих препаратов [10]. Перед проведением БДТ необходимо прекратить прием любых бронходилататоров согласно длительности их действия [19]. Единого стандарта в выборе бронходилататора на сегодняшний день нет. Рекомендуется использовать короткодействующие препараты для достижения максимального эффекта, например сальбутамол в дозе 400 мкг или фенотерол в той же дозе 
с использованием спейсера и соблюдением правил техники ингаляции. Если в качестве бронходилататора используется М-холинолитик, максимальная разовая доза составляет 160 мкг. Повторное исследование проводится через 15 мин после ингаляции $\beta 2$-агониста и через 30 мин после ингаляции М-холинолитика. Проба считается положительной, если после ингаляции $\beta 2$ агониста абсолютный прирост составляет 200 мл, а относительный - более $12 \%$.

Для расчета используются следующие формулы:

для расчета абсолютного прироста

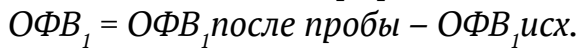

для расчета относительного прироста

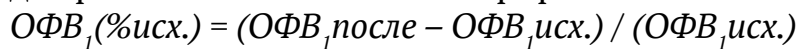

К БКТ относится тест с метахолином (гистамином), который может быть выполнен для выявления преходящей обструкции, а также БКТ с дозированной физической нагрузкой. Наиболее распространенным критерием обратимости обструкции является прирост ОФВ1 на 10\% по сравнению с исходным после физической нагрузки.

Во время проведения занятия по спирометрии следует подробно разобрать вопросы оценивания её показателей и построения заключения на основании рекомендаций Американского торакального общества/Европейского респираторного общества (ATS/ERS) 2005 г. [14] и Российского респираторного общества 2014 г. [10]. Необходимо провести анализ полученных данных, определить тип вентиляционных нарушений по основным параметрам, степень их тяжести, сформировать заключение, исходя из полученных данных. Также важным этапом является оценка правильности выполнения маневров, так как ошибки при их выполнении могут серьезно исказить результат, что, в свою очередь, может повлиять на выбор лечебной тактики. Обучение медицинского персонала навыкам контроля за проведением спирометрии является принципиально важным, чтобы не допустить диагностических ошибок. Если не удается выполнить пробу, необходимо дать пациенту время на отдых или повторить исследование в другой день.

В конце занятия для укрепления полученных знаний необходимо разобрать спирометрическое исследование на примерах заключений, выполненных реальным пациентам, после чего проводится итоговый тестовый контроль.

После несколько лет преподавания спирометрического метода диагностики на нашей кафедре терапии ФИПО им. проф. А.И. Дядыка мы пришли к выводу о том, что врачи проявляют повышенный интерес к этому исследованию. Следует отметить, что более качественному пониманию основ спирометрии помогает повторение анатомо-физиологических особенностей дыхательной системы, расширенное объяснение ключевых параметров респираторного цикла (ЖЕЛ, ФЖЕЛ, ОФВ1, ОФВ1/ЖЕЛ, ОФВ1/ФЖЕЛ), кривых поток-объем и поток-время, рассмотрение особенностей расшифровки полученных данных, в частности значение индекса Генслера, а также возможность непосредственно поучаствовать в ходе исследования, отработав на практике полученные знания. К педагогическим достоинствам изучаемой темы можно отнести возможность демонстрации всех этапов проведения спирометрии, включая настройку прибора, анализ спирометрических заключений с различными вариантами нарушений вентиляционной способности легких. Занятия по изучению спирометрического метода, как правило, вызывают живой интерес у аудитории с возможностью интерактивного обсуждения как полученных результатов, так и клинических аспектов тактики ведения больного.

\section{З А К ЛЮ Ч Е И Е}

Спирометрия - быстро выполняемый и надежный метод диагностики заболеваний легких на ранних стадиях с возможностью проведения исследования у постели больного, что особенно важно для своевременного лечения, предупреждения прогрессирования и профилактики осложнений. Преподавание основ спирометрического метода является необходимым и востребованным элементом подготовки высококвалифицированных специалистов, способствуя повышению качества медицинской помощи, быстрому принятию решений и выработке тактики ведения пациента.

Применение во время учебного процесса различных средств визуального представления (мультимедийное сопровождение, примеры заключений, графиков и кривых, тренировка с использованием аппаратуры и др.) повышает уровень восприятия и усвоения материала, улучшает качество образования слушателей ФИПО. Опыт внедрения основ спирометрии на кафедре терапии ФИПО им. проф. А.И. Дядыка показал, что большинство слушателей проявляют значительный интерес и стремление к восприятию преподаваемой информации, необходимой им в практической деятельности. 


\section{А.А. Калуга, Г.Г. Тарадин, И.В. Ракитская, К.Э. Могилевская, О.А. Приколота, И.С. Маловичко}

ГОО ВПО «Донецкий национальный медицинский университет имени М. Горького», Донецк

\section{ОСОБЕННОСТИ ПРЕПОДАВАНИЯ СПИРОМЕТРИЧЕСКОГО МЕТОДА ВРАЧАМ В ПОСЛЕДИПЛОМНОМ ОБУЧЕНИИ}

В статье рассматриваются особенности преподавания спирометрического метода на кафедре терапии факультета интернатуры и последипломного образования им. проф. А.И. Дядыка ГОО ВПО ДОННМУ ИМ. М.ГОРЬКОГО. Кратко описывается история создания спирографии. Подчеркиваются преимущества этого метода диагностики и актуальность его изучения врачами в последипломном обучении. Особое внимание уделяется вопросам преподавания этого метода слушателям, включая обсуждение подготовки к исследованию, технических особенностей аппара- туры, основных показаний и противопоказаний, этапов проведения исследования и ключевых оцениваемых параметров. Детально освещаются варианты интерпретации результатов, полученных в ходе исследования. Отдельно рассматривается обучение основам проведения бронходилатационного и бронхоконстрикторных тестов.

Ключевые слова: спирометрия, хроническая обструктивная болезнь легких, астма, функция внешнего дыхания, последипломное образование, обучение спирометрии, педагогика.

\section{A.A. Kaluga, G.G. Taradin, I.V. Rakitskaya, K.E. Mogilevskaia, O.A. Prikolota, I.S. Malovichko}

SEI HPE «M. Gorky Donetsk National Medical University», Donetsk

\section{SPECIFICS OF TEACHING OF THE SPIROMETRIC METHOD TO PHYSICIANS IN POSTGRADUATE EDUCATION}

The article discusses features of teaching of the spirometric method at the Department of Therapy named after prof. A. I. Dyadyk, Division of Internship and Postgraduate Training of the State Educational Organization of Higher Professional Education "M. Gorky Donetsk National Medical University". The history of spirography creation is briefly described. The advantages of this method of diagnosis and the relevance of its study by physicians in postgraduate training are emphasized. Special attention is paid to the preparation for the investigation, the study of technical features of the equipment, main indications and contraindications, the stages of the study and key parameters to be evaluated. Variants of interpretation of results obtained in the course of studies are described in detail. The basics of performing of bronchodilatatory and bronchoconstrictor tests are considered additionally.

Key words: spirometry, chronic obstructive pulmonary disease, asthma, respiratory function, postgraduate education, spirometry training, guidance.

\section{ЛИТЕРАТУРА}

1. Перельман Ю.М., Приходько А.Г. Спирографическая диагностика нарушений вентиляционной функции легких: Пособие для врачей: Издание 2-е, доп. Благовещенск; 2013. 44.

2. Steenbruggen I., Mitchell S., Cooper B. ERS European Spirometry Train-the-Trainer programme: good trainers are made, not born. Breathe. 2012; 8 (4): 263-266. doi:10.1183/20734735.012912

3. Global Strategy for the Diagnosis, Management and Prevention of Chronic Obstructive Lung Disease 2020. Global Initiative for Chronic Obstructive Lung Disease (GOLD). 2020. URL: https://goldcopd.org/wp-content/ uploads/2019/12/GOLD-2020-FINAL-ver1.2-03Dec19_ WMV.pdf

4. Global Initiative for Asthma (GINA). Global Strategy for Asthma Management and Prevention, Updated. 2020. URL: https://ginasthma.org/wp-content/uploads/2020/04/ GINA-2020-full-report_-final-_wms.pdf

5. Стручков П.В., Дроздов Д.В., Лукина О.Ф. Спирометрия: Руководство для врачей. М.; 2017. 96.

6. Hutchinson J. On the capacity of the lungs, and on the respiratory functions, with a view of establishing a precise and easy method of detecting disease by the spirometer. Med Chir Trans. 1846; 29: 137-252.

\section{REFERENCES}

1. Perel'man Yu.M., Prikhod'ko A.G. Spirograficheskaya diagnostika narushenii ventilyatsionnoi funktsii legkikh: Posobie dlya vrachei: Izdanie 2-e, dop. Blagoveshchensk; 2013. 44 (in Russian).

2. Steenbruggen I., Mitchell S., Cooper B. ERS European Spirometry Train-the-Trainer programme: good trainers are made, not born. Breathe. 2012; 8 (4): 263-266. doi:10.1183/20734735.012912

3. Global Strategy for the Diagnosis, Management and Prevention of Chronic Obstructive Lung Disease 2020. Global Initiative for Chronic Obstructive Lung Disease (GOLD). 2020. URL: https://goldcopd.org/wp-content/ uploads/2019/12/GOLD-2020-FINAL-ver1.2-03Dec19 WMV.pdf

4. Global Initiative for Asthma (GINA). Global Strategy for Asthma Management and Prevention, Updated. 2020. URL: https://ginasthma.org/wp-content/uploads/2020/04/ GINA-2020-full-report_-final-_wms.pdf

5. Struchkov P.V., Drozdov D.V., Lukina O.F. Spirometriya: Rukovodstvo dlya vrachei. M.; 2017. 96 (in Russian).

6. Hutchinson J. On the capacity of the lungs, and on the respiratory functions, with a view of establishing a precise and easy method of detecting disease by the spirometer. Med Chir Trans. 1846; 29: 137-252. 
7. Fleisch A. Der Pneumotachograph; ein Apparat zur Geschwindigkeitsregistrierung der Atemluft. Pflügers Archiv für die Gesamte Physiologie des Menschen und der Tiere. 1925; 209: 713-722. doi: 10.1007/bf01730956

8. Dayman H. Mechanics of airflow in health and in emphysema. J Clin Invest. 1951; 30 (11): 1175-1190. doi: 10.1172/ JCI102537

9. Wright B.M., McKerrow C.B. Maximum forced expiratory flow rate as a measure of ventilatory capacity: with a description of a new portable instrument for measuring it. Br Med J. 1959; 2 (5159): 1041-1046. doi: 10.1136/ bmj.2.5159.1041

10. Чучалин А.Г., Айсанов 3.Р., Чикина С.Ю., Черняк А.В., Калманова Е.Н. Федеральные клинические рекомендации Российского респираторного общества по использованию метода спирометрия. Пульмонология. 2014; 6: 11-24. doi: 10.18093/0869-0189-2014-0-6-11-24

11. Miller M.R., Crapo R., Hankinson J. et al. General considerations for lung function testing. Eur Respir J. 2005; 26 (1): 153-161. doi: 10.1183/09031936.05.00034505

12. Cooper B.G. An update on contraindications for lung function testing. Thorax. 2011; 66: 714-723. doi: 10.1136/ thx.2010.139881

13. Miller M.R., Hankinson J., Brusasco V., et al. Standardisation of spirometry. Eur Respir J. 2005; 26 (2): 319-338. doi: 10.1183/09031936.05.00034805

14. Pellegrino R., Viegi G., Brusasco V. et al. Interpretative strategies for lung function tests. Eur Respir J. 2005; 26 (5): 948-968. doi: 10.1183/09031936.05.00035205

15. Клемент Р.Ф., Лаврушин А.А., Котегов Ю.М., ТерПогасян П.А. Инструкция по применению формул и таблиц должных величин основных спирографических показателей. Л.; 1986. 79.

16. Савельев Б.П., Ширяева И.С. Функциональные параметры системы дыхания у детей и подростков. М.: 2001. 232.

17. Knudson R.J., Lebowitz M.D., Holberg C.J., Burrows B. Changes in the normal maximal expiratory flow-volume curve with growth and aging. Am Rev Respir Dis. 1983; 127 (6): 725-734. doi: 10.1164/arrd.1983.127.6.725

18. Руководство по клинической физиологии дыхания. Под ред. Шика Л.Л., Канаева Н.Н. М.; 1980. 374.

19. Ватутин Н.Т., Смирнова А.С., Тарадин Г.Г. Современные доставочные устройства при лечении хронических обструктивных заболеваний легких. Пульмонология. 2015; 25 (4): 477-482. doi: 10.18093/0869-0189-2015$25-4-477-482$
7. Fleisch A. Der Pneumotachograph; ein Apparat zur Geschwindigkeitsregistrierung der Atemluft. Pflügers Archiv für die Gesamte Physiologie des Menschen und der Tiere. 1925; 209: 713-722. doi: 10.1007/bf01730956

8. Dayman H. Mechanics of airflow in health and in emphysema. J Clin Invest. 1951; 30 (11): 1175-1190. doi: 10.1172/ JCI102537

9. Wright B.M., McKerrow C.B. Maximum forced expiratory flow rate as a measure of ventilatory capacity: with a description of a new portable instrument for measuring it. Br Med J. 1959; 2 (5159): 1041-1046. doi: 10.1136/ bmj.2.5159.1041

10. Chuchalin A.G., Aisanov Z.R., Chikina S.Yu., Chernyak A.V., Kalmanova E.N. Federal'nye klinicheskie rekomendatsii Rossiiskogo respiratornogo obshchestva po ispol'zovaniyu metoda spirometriya. Pul'monologiya. 2014; 6: 11-24 (in Russian). doi: 10.18093/0869-01892014-0-6-11-24

11. Miller M.R., Crapo R., Hankinson J. et al. General considerations for lung function testing. Eur Respir J. 2005; 26 (1): 153-161. doi: 10.1183/09031936.05.00034505

12. Cooper B.G. An update on contraindications for lung function testing. Thorax. 2011; 66: 714-723. doi: 10.1136/ thx.2010.139881

13. Miller M.R., Hankinson I., Brusasco V., et al. Standardisation of spirometry. Eur Respir J. 2005; 26 (2): 319-338. doi: 10.1183/09031936.05.00034805

14. Pellegrino R., Viegi G., Brusasco V. et al. Interpretative strategies for lung function tests. Eur Respir J. 2005; 26 (5): 948-968. doi: 10.1183/09031936.05.00035205

15. Klement R.F., Lavrushin A.A., Kotegov Yu.M., Ter-Pogasyan P.A. Instruktsiya po primeneniyu formul i tablits dolzhnykh velichin osnovnykh spirograficheskikh pokazatelei. L.; 1986. 79 (in Russian).

16. Savel'ev B.P., Shiryaeva I.S. Funktsional'nye parametry sistemy dykhaniya u detei i podrostkov. M.: 2001. 232 (in Russian).

17. Knudson R.J., Lebowitz M.D., Holberg C.J., Burrows B. Changes in the normal maximal expiratory flow-volume curve with growth and aging. Am Rev Respir Dis. 1983; 127 (6): 725-734. doi: 10.1164/arrd.1983.127.6.725

18. Rukovodstvo po klinicheskoi fiziologii dykhaniya. Pod red. Shika L.L., Kanaeva N.N. M.; 1980. 374 (in Russian).

19. Vatutin N.T., Smirnova A.S., Taradin G.G. Sovremennye dostavochnye ustroistva pri lechenii khronicheskikh obstruktivnykh zabolevanii legkikh. Pul'monologiya. 2015; 25 (4): 477-482 (in Russian). doi: 10.18093/0869-01892015-25-4-477-482. 